\title{
EFECTIVIDAD DE LA HIPEROXIGENACIÓN MATERNA Y TOCOLISIS AGUDA EN MONITOREO ELECTRÓNICO FETAL INTRAPARTO ALTERADO
}

\author{
Felipe Núñez V. ${ }^{1}$, Jorge Carvajal C. PhD ${ }^{2}$
}

\footnotetext{
${ }^{1}$ Servicio de Obstetricia y Ginecología, Hospital Dr. Sótero del Río. ${ }^{2}$ Departamento de Obstetricia y Ginecología, Unidad de Medicina Materno Fetal, Facultad de Medicina, Pontificia Universidad Católica de Chile.
}

\section{RESUMEN}

El trabajo de parto es un evento de riesgo para hipoxia fetal aguda, lo que puede detectarse en alteraciones del Monitoreo Electrónico Fetal Intraparto (MEFI). Frente a un MEFI sugerente de hipoxemia fetal, existen maniobras estándar de resucitación intrauterina como lateralización de la paciente, aporte de volumen intravenoso y suspensión de la infusión oxitócica. Se debate la utilidad de la hiperoxigenación materna y la tocolisis aguda. Revisamos la evidencia científica relevante sobre la utilidad y seguridad de estas dos maniobras. Encontramos sólo limitada evidencia respecto del efecto benéfico de la hiperoxigenación materna frente a alteraciones del MEFI, existiendo dudas de su inocuidad. La tocolisis aguda ha demostrado ser eficaz en lograr la normalización del MEFI, independiente de la presencia de hiperactividad uterina, con una efectividad cercana al $80 \%$. La evidencia apoya el uso de ß-miméticos y Nitroglicerina. Recomendamos incluir en la práctica clínica habitual la tocolisis aguda y la hiperoxigenación materna sólo por lapsos breves, junto a las maniobras habituales de resucitación intrauterina frente a un MEFI alterado.

\section{PALABRAS CLAVE: Monitoreo electrónico fetal intraparto, hipoxia fetal, hiperoxigenación materna, tocolisis intraparto, resucitación intrauterina}

\section{SUMMARY}

Labor is a risk condition for acute fetal hypoxia, this hypoxia can be detected by using cardiotocography (CTG). When CTG suggest hypoxia, intrauterine resuscitation techniques must be implemented, such as lateral positioning of the mother, intravenous fluid administration and suspension of oxytocin administration. Among intrauterine resuscitation techniques it is discussed the use of maternal hyperoxygenation and acute tocolysis. Here we review the evidence supporting utility and safety of these two techniques. We found only limited evidence supporting the beneficial role of maternal hyperoxygenation after nonreassuring CTG, plus concerns about its safety. Acute tocolysis has proven to be efficient in normalizing CTG, independent of the presence of uterine hyperactivity, with an overall benefit of $80 \%$. Evidence supports the use of B-mimetics and nitroglycerin. We recommend to include acute tocolysis and maternal hyperoxygenation (just for limited time), among intrauterine resuscitation techniques for abnormal CTG.

KEY WORDS: Cardiotocography, fetal hypoxia, maternal hyperoxygenation, acute tocolysis, intrauterine resuscitation 


\section{INTRODUCCIÓN}

Durante el trabajo de parto existe riesgo de hipoxia aguda y acidosis fetal (1), por lo que se han desarrollado múltiples métodos de evaluación del bienestar fetal intraparto, tales como: auscultación intermitente de latidos cardiacos fetales (LCF), monitoreo electrónico fetal intraparto (MEFI), estimulación vibroacústica fetal, oximetría de pulso fetal, medición de $\mathrm{pH}$ de cuero cabelludo y electrocardiografía (ECG) fetal intraparto (2).

El método más utilizado para la evaluación del bienestar fetal intraparto es el MEFI, método evaluado en una revisión sistemática de la base de datos Cochrane en el año 2006 (3), que mostró que el uso de MEFI disminuye la tasa de convulsiones neonatales en un $50 \%$, sin modificar la tasa de parálisis cerebral ni la mortalidad perinatal, en esta revisión se observó también un aumento significativo de la tasa de cesáreas y partos vaginales operatorios asociados al uso de MEFI. Pese a su limitada utilidad, el MEFI se ha convertido en el método de elección de evaluación del bienestar fetal intraparto a nivel mundial, dado su fácil implementación.

Se ha definido como bienestar fetal en el MEFI la presencia de 4 criterios: frecuencia cardiaca fetal basal entre 110 y 160 latidos por minuto (lpm), variabilidad entre 5-25 lpm, ausencia de desaceleraciones y presencia de aceleraciones (no siendo estas indispensables). Cualquier alteración de estos parámetros se considera como registro anormal o MEFI alterado según lo estipulado en las guías clínicas de la National Institute of Child Health and Human Development (NICHD) (4), el American College of Obstetricians and Gynecologists (ACOG) (5) y el Royal College of Obstetricians and Gynae- cologists (RCOG) (6). El registro anormal se puede clasificar en sospechoso u ominoso según se detaIla en las Tablas I y II (6).

Ante un registro anormal existen diversas maniobras descritas para lograr un registro de bienestar fetal, a esto se le llama "resucitación intrauterina". Algunas de las maniobras de resucitación intrauterina, como la lateralización materna, suspensión de infusión oxitócica y el aporte de volumen intravenoso, son ampliamente avaladas por la literatura; otras, como la hiperoxigenación materna y la tocolisis intraparto, son consideradas como parte del manejo, pero existe debate respecto de su real utilidad y seguridad.

El objetivo de este documento es revisar específicamente estas dos maniobras de resucitación intrauterina ante un MEFI alterado: hiperoxigenación materna y tocolisis intraparto, para luego elaborar una recomendación de manejo basada en la mejor evidencia disponible.

\section{HIPEROXIGENACIÓN MATERNA}

La gran mayoría de las pacientes en trabajo de parto tienen saturación de oxígeno normal (mayor a $95 \%$ ), por lo que la administración de oxígeno no debiera cambiar significativamente la saturación de oxígeno materna. Sin embargo, aumentaría sustancialmente la presión arterial de oxígeno a expensas del oxígeno soluble, produciendo un leve aumento en la concentración plasmática de oxígeno. En teoría, si la perfusión placentaria y el área de intercambio gaseoso en el espacio intervelloso no se encuentran alterados, esto se traduciría en una mayor difusión de oxígeno hacia el territorio fetal, reduciendo así la hipoxia y acidosis $(7,8)$.

Tabla I

MONITOREO ELECTRÓNICO FETAL INTRAPARTO: ANÁLISIS DE LAS VARIABLES

\begin{tabular}{|c|c|c|c|c|}
\hline Registro & $\begin{array}{l}\text { FCFB } \\
(\mathrm{lpm})\end{array}$ & $\begin{array}{l}\text { Variabilidad } \\
\text { (lpm) }\end{array}$ & Desaceleraciones & Aceleraciones \\
\hline Tranquilizador & $110-160$ & 5 & Ausentes & Presentes \\
\hline No & $100-109$ & $<5$ por & Precoces & - \\
\hline tranquilizador & $161-180$ & $\begin{array}{l}40 \mathrm{~min} \\
\text { pero }<90 \mathrm{~min}\end{array}$ & $\begin{array}{l}\text { Variables simples } \\
\text { Única } 3 \mathrm{~min}\end{array}$ & \\
\hline \multirow[t]{2}{*}{ Anormal } & $<100$ & $<5 \mathrm{lpm}$ & Variables complejas & - \\
\hline & $\begin{array}{l}>180 \\
\text { Sinusoidal }\end{array}$ & por $90 \mathrm{~min}$ & $\begin{array}{l}\text { Tardías } \\
\text { Única > } 3 \text { min }\end{array}$ & \\
\hline
\end{tabular}

FCFB: Frecuencia Cardiaca Fetal Basal. Ipm: latidos por minuto. 
Tabla II

\section{MONITOREO ELECTRÓNICO FETAL INTRAPARTO: INTERPRETACIÓN DEL TRAZADO}

\begin{tabular}{ll}
\hline Registro & Interpretación \\
\hline Normal & $\begin{array}{l}\text { Los 4 parámetros están en rango } \\
\text { tranquilizador }\end{array}$ \\
Sospechoso & $\begin{array}{l}\text { Uno de los parámetros cae en } \\
\text { rango no tranquilizador }\end{array}$ \\
Patológico & $\begin{array}{l}\text { Dos parámetros caen en rango } \\
\text { no tranquilizador o un parámetro } \\
\text { cae en rango anormal }\end{array}$ \\
\hline
\end{tabular}

Si la hiperoxigenación materna mejora la oxemia fetal y revierte las alteraciones del MEFI ha sido motivo de intenso debate durante los últimos 40 años. A continuación se analizan los efectos biológicos y clínicos de la hiperoxigenación materna.

Beneficios de la hiperoxigenación materna: Estudios prospectivos muestran que la administración de alto flujo de oxígeno a la madre en trabajo de parto, mejora la saturación de $\mathrm{O}_{2}$ fetal $\left(\mathrm{SaO}_{2} \mathrm{~F}\right)$ entre un 5 y $10 \%$, medido por oximetría de pulso, siendo mayor el beneficio a mayor fracción inspirada de oxígeno y en fetos con $\mathrm{SaO}_{2} \mathrm{~F}$ basal más baja. Este aumento ocurre frente a MEFI normal $(9,10)$ y anormal (11). Así mismo, un estudio realizado con espectroscopia infrarroja en fetos en trabajo de parto no complicado, mostró que la hiperoxigenación materna aumentó significativamente la concentración plasmática de oxihemoglobina y la saturación de oxígeno cerebral de un $44 \%$ a un $57 \%(p<0,001)$ (12).

Otro estudio prospectivo evaluó 34 fetos con restricción de crecimiento intrauterino (RCIU) y 35 fetos con peso adecuado para la edad gestacional (AEG), todos cercanos al término. Se registraron lo cambios en el registro basal no estresante (RBNE) antes, durante y después de administrar alto flujo de oxígeno materno $(10 \mathrm{l} / \mathrm{min})$. Los resultados de este estudio mostraron que durante la administración de oxígeno materno se produce un aumento del número de aceleraciones en el RBNE del $30 \%$, tanto en fetos RCIU como AEG $(p<0,001)$, y un aumento significativo de la variabilidad $(p<0,001)(13)$.

Respecto de la hiperoxigenación materna en MEFI alterado, un estudio randomizado controlado, efectuado en 36 primates, demostró que el uso de oxígeno materno frente a MEFI alterado logra una abolición o disminución significativa de las desaceleraciones tardías en casi todos los fetos (14). Dos estudios prospectivos clásicos, realizados en hu- manos, observaron el efecto de la hiperoxigenación materna en el MEFI.

El primer estudio (15), evaluó 21 pacientes en trabajo de parto, a las cuales se administró oxígeno en condiciones de bienestar fetal y en MEFI alterado. Los resultados de este estudio mostraron que en condiciones de bienestar fetal no se alteran las variables del MEFI; en fetos con taquicardia: la frecuencia cardiaca fetal basal (FCFB) disminuyó significativamente en todos los casos (5-30 lpm); en todos aquellos fetos que presentaron desaceleraciones tardías: la amplitud de las desaceleraciones tardías disminuyó entre un 50 y un $100 \%$.

El segundo estudio (16), evaluó en 20 fetos de término en trabajo de parto el efecto de la hiperoxigenación materna en la $\mathrm{SaO}_{2} \mathrm{~F}$ y estado ácidobase. Como hallazgo, los autores observaron, en aquellos fetos que presentaron desaceleraciones tardías en el MEFI, una abolición o disminución de la frecuencia de las desaceleraciones tardías en respuesta a la hiperoxigenación. Este estudio no posee un análisis estadístico de esta observación por no ser el objetivo primario del estudio.

Una revisión de la base de datos Cochrane en el año 2003 (17), concluyó que no existen estudios randomizados controlados que evalúen la efectividad clínica de la hiperoxigenación materna como método de resucitación intrauterina ante un MEFI alterado.

Riesgos de la hiperoxigenación materna: Dos estudios randomizados controlados evaluaron el uso profiláctico de oxígeno en trabajo de parto. El primer estudio (18), utilizó oxígeno materno profiláctico durante el período expulsivo, en 86 pacientes con trabajo de parto normal, evidenciando un mayor número de recién nacidos con $\mathrm{pH}$ de arteria umbilical $<7,2$ en el grupo de hiperoxigenación materna. Este deterioro fue observado sólo en aquellos niños sometidos a oxígeno por más de 10 minutos. El segundo estudio (19), utilizó la misma metodología para 160 pacientes, encontrando un mayor número de gases de cordón con $\mathrm{pH}<7,2$ en el grupo intervenido, independiente del tiempo de duración de la hiperoxigenación materna.

Estos dos estudios motivaron preocupación por las posibles consecuencias adversas de la hiperoxigenación materna durante el trabajo de parto. Consideramos, sin embargo, que estos datos no son extrapolables a fetos con MEFI alterado, dado que ambos estudios fueron realizados en fetos con trabajo de parto normal, el oxígeno fue utilizado durante el período expulsivo y de manera profiláctica, lo que difiere del uso que estudiamos.

Sin embargo, existe evidencia de que la hiperoxigenación materna aumenta la disponibilidad de 
radicales libres tanto en sangre materna como fetal (20). La importancia clínica de este hallazgo aún no ha sido esclarecida, pero creemos que si debe ser tomada en consideración, y limitar la hiperoxigenación a tiempos breves e indispensables del trabajo de parto (21).

\section{TOCOLISIS INRAPARTO}

Durante el trabajo de parto se reduce la oxigenación fetal (1), principalmente durante las contracciones uterinas, momento durante el cual la $\mathrm{SaO}_{2} \mathrm{~F}$ disminuye en promedio un $7 \%$, pudiendo mantenerse este descenso hasta 2 minutos poscontracción (22). La polisistolia (contracciones uterinas $>5$ en 10 minutos) no le permite al feto recuperar su estado de oxemia normal, de modo que el descenso de la $\mathrm{SaO}_{2} \mathrm{~F}$ es aún más marcado (18\%) (23). La hipoxia fetal secundaria a polisistolía por uso de oxitocina exógena, habitualmente se revierte con la suspensión de la oxitocina (23). Sin embargo, la polisistolia también puede presentarse en respuesta al uso de prostaglandinas como el Misoprostol o de manera espontánea (32), así la suspensión de la ocitocina no siempre es una alternativa. Es importante considerar, además, que puede haber hipoxia fetal con un patrón de contracciones uterinas normales en fetos con baja reserva de oxígeno (24).

El conocimiento de los cambios en la $\mathrm{SaO}_{2} \mathrm{~F}$ asociado a las contracciones uterinas llevó a0 postular el uso de tocolíticos intraparto, para lograr una relajación uterina inducida farmacológicamente y mejorar así la oxigenación fetal, en casos en que se sospeche deterioro de la oxigenación, sea en presencia o no de polisistolía.

Desde que Caldeyro-Barcia sugiriera el uso de tocolisis intraparto como maniobra de resucitación intrauterina en los años 60 (25), han aparecido múltiples estudios para evaluar su efectividad. En 1998, Kulier y Hofmeyr (26), llevaron a cabo una revisión sistemática en la base de datos Cochrane, considerando 3 estudios randomizados controlados que utilizaron $\beta$-miméticos como maniobra de resucitación intrauterina. El primer estudio (27), comparó Terbutalina vs no tratamiento, mejorando el MEFI en el $91 \%$ de las pacientes tratadas con Terbutalina y $0 \%$ en el grupo de no tratamiento (RR 11,11; IC 95\% 1,7-100). El segundo estudio (28), comparó Terbutalina vs Sulfato de Magnesio, logrando mejoría del MEFI en $91,3 \%$ de las pacientes tratadas con Terbutalina y $69,6 \%$ de las pacientes tratadas con Sulfato de Magnesio. Esta diferencia no fue estadísticamente significativa (RR 3,45; IC $95 \%$ 0,81-14,3). El tercer estudio (29), comparó Hexoprenaline vs no tratamiento, siendo Hexopre- naline superior, con una tasa de mejoría del MEFI de $61,6 \%$ vs $10 \%$ en el grupo sin tratamiento (RR 2,33; IC 95\% 1,14-4,76). La conclusión de esta revisión sistemática fue que ante MEFI alterado, los - -miméticos como maniobra de resucitación intrauterina son superiores a no tratamiento, con una tasa de éxito del 75\% (RR 3,85; IC 95\% 1,89-7,7). Sin embargo, a pesar de estos resultados, los autores concluyen que no hay evidencia suficiente para recomendar su uso cotidiano en la práctica clínica (26), dado la ausencia de cambios significativos en la morbimortalidad perinatal.

En 2007, Brioso y cols (30), conducen un estudio prospectivo, randomizado y controlado con 390 fetos con MEFI alterado, asignándolos aleatoriamente a interrupción inmediata (parto, cesárea o fórceps) vs tocolisis con Fenoterol y parto diferido. El resultado de este estudio mostró que la tocolisis con Fenoterol tuvo una efectividad de $81,3 \%$ en mejorar el MEFI, no hubo diferencias en el pH de sangre de cordón $<7,1$, Apgar 1' $\leq 3$ ni Apgar 5' <7; el grupo de interrupción de emergencia tuvo un mayor número de recién nacidos con exceso de base <12 (RR 1,48; IC 95\% 1,00-2,20), y una mayor probabilidad de admisión a $\mathrm{UCl}$ neonatal (RR 2,14 ; IC 95\% 1,23-3,74). Cabe destacar que en el grupo asignado a tocolisis hubo un porcentaje significativamente mayor de operación cesárea $(90 \%$ vs. $80 \%$ en el grupo de interrupción inmediata; RR 1,63 , IC $95 \% 1,10-2,42$ ).

Un estudio evaluó en 22 pacientes la eficacia de la Nitroglicerina como tocolítico agudo ante diferentes emergencias obstétricas no relacionadas con el MEFI, logrando una relajación uterina satisfactoria en el $100 \%$ de los casos (31). Otro estudio evaluó la efectividad de Nitroglicerina en 24 pacientes con MEFI alterado que no respondió a lateralización de la paciente, hiperoxigenación materna y suspensión de oxitocina al cabo de 2-5 minutos. Se observó que el uso de bolos de Nitroglicerina $(60-90 \mu \mathrm{g}$ iv) mejoró el MEFI en el 100\% de los casos en un lapso de 4-5 minutos. Se reportó como efecto adverso hipotensión en el $25 \%$ de las pacientes $(p<0,0009)$ que se resolvió en todos los casos con pequeñas dosis de efedrina (4,5-6 mg iv) (32).

Respecto del tipo de tocolítico a usar ante un MEFI alterado, 2 estudios randomizados han comparado la utilidad de diferentes tocolíticos como medida de resucitación intrauterina. El primer estudio (33), comparó Atosiban vs Hexoprenaline en 26 pacientes con bradicardia fetal, logrando una normalización del MEFI en el $92 \%$ y $100 \%$ de los casos respectivamente (diferencia no significativa). El segundo estudio (34), comparó Terbutalina vs Nitroglicerina en 110 pacientes con MEFI alterado, 
normalizando el MEFI en $72 \%$ y $64 \%$, respectivamente (diferencia no significativa).

Consideramos que no existe evidencia suficiente para recomendar un tipo de tocolítico por sobre otro, todas las drogas usadas muestran eficacias similares y superiores al $60 \%$. Las diferencias fundamentales radican en los efectos secundarios de cada tocolítico, principalmente hipotensión asociada al uso de Nitroglicerina $(32,34)$ y taquicardia asociada al uso de ß-miméticos $(34,35)$.

Es también importante notar que, los resultados de los estudios sobre la efectividad de la tocolisis intraparto, como maniobra de resucitación intrauterina, frente a un MEFI alterado, son similares independiente de si la hiperactividad uterina fue considerada como criterio de inclusión (32), o no fue considerada $(30,33,34)$.

\section{DISCUSIÓN}

En este artículo revisamos la hiperoxigenación materna y tocolisis intraparto como maniobras de resucitación intrauterina ante un MEFI alterado, sus fundamentos biológicos y utilidad clínica, así como su efectividad, y efectos adversos y riesgos potenciales.

Respecto de la hiperoxigenación materna como maniobra de resucitación intrauterina, es clara la lógica biológica que sustenta su uso. Los estudios realizados muestran un beneficio de la hiperoxigenación materna en la saturometría de pulso fetal, RBNE y en mejorar el MEFI alterado (9-12). Sin embargo, la escasa evidencia existente en este punto y sus problemas metodológicos, no permiten sacar conclusiones definitivas respecto de su real beneficio (17), haciendo notoria la necesidad de desarrollar estudios prospectivos randomizados controlados, de buena calidad metodológica, que resuelvan el debate de si la hiperoxigenación materna es una medida efectiva de resucitación intrauterina.

Del mismo modo, no se ha demostrado la seguridad de la hiperoxigenación materna. Las dudas respecto de su inocuidad se basan en estudios con diseños hechos en poblaciones no representativas de hipoxia fetal intraparto $(18,19)$, por lo que no son extrapolables a fetos con MEFI alterado. Hasta que aparezca nueva evidencia que resuelva la controversia del riesgo-beneficio de la hiperoxigenación materna, frente a un MEFI alterado, en conjunto con las otras maniobras de resucitación intrauterina parece razonable el uso de la hiperoxigenación materna por períodos limitados.

Respecto de la tocolisis intraparto, hemos analizado la importancia de la contracción uterina en el desarrollo de hipoxia fetal intraparto, ya sea asocia- do a polisistolía o a un patrón normal de contracciones uterinas. No extraña pues, que al producir una relajación uterina farmacológica, el feto pueda recuperar su estado de oxemia basal y mejorar así las alteraciones que se produzcan en el MEFI. Hay suficiente evidencia para afirmar que la tocolisis intraparto, es una maniobra de resucitación intrauterina efectiva, en mejorar las alteraciones del MEFI con una efectividad promedio cercana al $80 \%$ (27-29). Esta efectividad está claramente demostrada para B-miméticos y Nitroglicerina, no existiendo estudios suficientes que confirmen a Atosiban como tocolítico efectivo para resucitación intrauterina frente a un MEFI alterado.

La evidencia existente no muestra superioridad de un tocolítico respecto de otro, son pocos los estudios que comparan los diferentes tocolíticos, y los estudios existentes no han mostrado diferencias entre B-miméticos y Nitroglicerina, por lo que la elección del tocolítico debiera basarse en la experiencia del equipo clínico y los riesgos individualizados de cada paciente según los potenciales efectos adversos de cada droga.

Nuestra recomendación es que frente a un MEFI alterado, luego de la evaluación de las condiciones cervicales, se debe lateralizar a la paciente, aportar volumen intravenoso con soluciones isotónicas, suspender la infusión oxitócica (si corresponde), aportar oxígeno $(10 \mathrm{l} / \mathrm{min})$ por un lapso breve (hasta mejoría del MEFI) e iniciar tocolisis intraparto, independiente de si la alteración del MEFI está dada en contexto de una polisistolía o no. En nuestro centro utilizamos como tocolítico la Nitroglicerina (50-100 $\mu \mathrm{g}$ iv en bolo) dado su fácil administración y vida media corta.

\section{BIBLIOGRAFÍA}

1. Seelbach-Gobel B, Heupel M, Kuhnert M, Butterwegge $M$. The prediction of fetal acidosis by means of intrapartum fetal pulse oximetry. Am J Obstet Gynecol 1999;180:73-81.

2. Barrena N, Carvajal J. Evaluación fetal intraparto. Análisis crítico de la evidencia. Rev Chil Obstet Ginecol 2006;71(1):63-8.

3. Alfirevic Z, Devane D, Gyte GML. Continuous cardiotocography (CTG) as a form of electronic fetal monitoring (EFM) for fetal assessment during labour (Review). Cochrane Database Syst Rev. 2006;(3): CD006066.

4. National Institute of Child Health and Human Development Research Planning Workshop. Electronic fetal heart rate monitoring: Research guidelines for interpretation. Am J Obstet Gynecol 1997;177:1385-90.

5. American College of Obstetricians and Gynecologists. Intrapartum fetal heart rate monitoring. Practice bulletin No.62. Obstet Gynecol 2005;105(5):1161-9. 
6. Royal College of Obstetricians and Gynaecologists. The use of electronic fetal monitoring. The use and interpretation of cardiotocography in intrapartum fetal surveillance. Evidence-based Clinical Guideline Number 8. 2001. ISBN 1-900364-49-2. www.rcog.org.uk

7. Seeds E, Escarcena L. Prevention and correction of fetal acidosis and hypoxia. Clin Obstet Gynecol 1974;17(3):115-34.

8. Downing J, Brock-Utne J, Mankowitz M. Aspects of intra-uterine fetal resuscitation. S Afr Med J 1980;58(9):373-5.

9. Simpson K, James D. Efficacy of intrauterine resuscitation techniques in improving fetal oxygen status during labor. Obstet Gynecol 2005;105:1362-8.

10. Roztocil A, Miklica J, Ventubra P, Kucera M, Kachlik $P$. Effect of maternal $\mathrm{O} 2$ inhalation on oxygen saturation in the parturient (SpO2) and the fetus (FSpO2). Ceska Gynekol 2000;65(6):393-7.

11. Haydon M, Gorenberg D, Nageotte M, Ghamsary M, Rumney P, Patillo C. The effect of maternal oxygen administration on fetal pulse oximetry in labor in fetuses with nonreassuring fetal heart rate patterns. Am J Obstet Gynecol 2006;195:735-8.

12. Aldrich C, Wyatt J, Spencer J, Reynolds E, Delpy D. The effect of maternal oxygen administration on human fetal cerebral oxygenation measured during labour by near infrared spectroscopy. Br J Obstet Gynaecol 1994;101(6):509-13.

13. Bartnicki J, Saling E. Influence of maternal oxygen administration on the computer-analysed fetal heart rate patterns in small-for-gestational-age fetuses. Gynecol Obstet Invest 1994;37(3):172-5.

14. Morishima H, Daniel S, Richards R, James L. The effect of increased maternal $\mathrm{PaO} 2$ upon the fetus during labor. Am J Obstet Gynecol 1975;123(3):257-64.

15. Althabe J, Schwarcz R, Pose S, Escarcena L, Caldeyro-Barcia R. Effects on fetal heart rate and fetal pO2 of oxygen administration to the mother. Am J Obstet Gynecol 1967;98:858-70.

16. Khazin A, Hon E, Hehre F. Effects of maternal hyperoxia on the fetus. Am J Obstet Gynecol 1971;109:62837.

17. Fawole B, Hofmeyr G. Maternal oxygen administration for fetal distress. Cochrane Database Syst Rev 2003;(4): CD000136.

18. Thorp J, Trobough T, Evans R, Hedriek J, Yeast J. The effect of maternal oxygen administration during the second stage of labor on umbilical cord blood gas values: A randomized controlled prospective trial. Am J Obstet Gynecol 1995;172:465-74.

19. Sirimai K, Atisook R, Boriboonhirunsarn D. The correlation of intrapartum maternal oxygen administration and umbilical cord blood gas values. Acta Obstet Gynecol Scand Supp 1997;76(167:2):90.

20. Khaw K, Wang C, Ngan Kee W, Pang C, Rogers M. Effects of high inspired oxygen fraction during elective Caesarean section under spinal anaesthesia on ma- ternal and fetal oxygenetion and lipid peroxidation. $\mathrm{Br}$ J Anaesth 2002;88:18-23.

21. Simpson K. Intrauterine resuscitation during labor: Should maternal oxygen administration be a first-line measure? Semin Fetal Neonatal Med 2008;13(6):3627.

22. McNamara $\mathrm{H}$, Johnson $\mathrm{N}$. The effect of uterine contractions on fetal oxygen saturation. $\mathrm{Br} \mathrm{J}$ Obstet Gynaecol 1995;102:644-7.

23. Johnson N, van Oudgaarden E, Montague I, McNama$\mathrm{ra} \mathrm{H}$. The effect of oxytocin-induced hyperstimulation on fetal oxygen. Br J Obstet Gynaecol 1994;101:8057.

24. Thurlow J, Kinsella S. Intrauterine resuscitation: active management of fetal distress. Int J Obstet Anesth 2002;11(2):105-16.

25. Cowan D. Intrapartum fetal resuscitation. S Afr Med J 1980;58(9):376-9.

26. Kulier R, Hofmeyr G. Tocolytics for suspected intrapartum fetal distress. Cochrane Database Syst Rev 1998;(2):CD000035.

27. Patriarco M, Viechnicki B, Hutchinson T, Klasko $S$, Yeh S. A study on intrauterine fetal resuscitation with terbutaline. Am J Obstet Gynecol 1987;157:384-7.

28. Magann E, Cleveland R, Dockery J, Chauhan S, Martin J, Morrison J. Acute tocolysis for fetal distress: terbutaline versus magnesium sulphate. Aust $\mathrm{N} \mathrm{Z} \mathrm{J}$ Obstet Gynaecol 1993;4:362-6.

29. Kulier R, Gülmezoglu AM, Hofmeyr GJ, Van Gelderen CJ. Betamimetics in fetal distress: a randomised controlled trial. J Perinat Med 1997;25:97-100.

30. Briozzo L, Martinez A, Nozar M, Fiol V, Pons J, Alonso $\mathrm{J}$. Tocolysis and delayed delivery versus emergency delivery in cases of non-reassuring fetal status during labor. J Obstet Gynaecol Res 2007;33(3):266-73.

31. O'Grady J, Parker R, Patel S. Nitroglycerin for rapid tocolysis: development of a protocol and a literature review. J Perinatol 2000;1:27-33.

32. Mercier F, Dounas M, Bouaziz H, Lhuissier C, Benhamou $D$. Intravenous nitroglycerin to relieve intrapartum fetal distress relates to uterine hyperactivity: A prospective observational study. Anesth Analg 1997;84:1117-20.

33. Afschar P, Schöll W, Bader A, Bauer M, Winter R. A prospective randomised trial of atosiban versus hexoprenaline for acute tocolysis and intrauterine resuscitation. Br J Obstet Gynaecol 2004;111:316-8.

34. Pullen K, Riley E, Waller S, Taylor L, Caughey A, Druzin $\mathrm{M}$, et al. Randomized comparison of intravenous terbutaline vs nitroglycerin for acute intrapartum fetal resuscitation. Am J Obstet Gynecol 2007;197:414. e1-414.e6.

35. De Heus R, Mulder E, Derks J, Kurver P, Van Wolfswinkel L, Visser G. A prospective randomized trial of acute tocolysis in term labour with atosiban or ritodrine. Eur J Obstet Gynecol Reprod Biol 2008;139(2):13945. 